\title{
Analysis of Endoscopic Sphenopalatine Artery Surgery for Refractory Epistaxis
}

\author{
Takahiro Kitamura*, Yukinori Takenaka, Kengo Aoki, Takuma Shimada, Hiroko Hamaguchi, \\ Ayaka Nakatani, Masayuki Nozawa, Ryohei Oya, Yoshifumi Yamamoto, Atsuhiko Uno \\ Department of Otorhinolaryngology-Head and Neck Surgery, Osaka General Medical Center, Osaka, Japan \\ Email: *ent25kitamura@gmail.com
}

How to cite this paper: Kitamura, T., Takenaka, Y., Aoki, K., Shimada, T., Hamaguchi, H., Nakatani, A., Nozawa, M., Oya, R., Yamamoto, Y. and Uno, A. (2020) Analysis of Endoscopic Sphenopalatine Artery Surgery for Refractory Epistaxis. International Journal of Otolaryngology and Head \& Neck Surgery, 9, 78-85.

https://doi.org/10.4236/ijohns.2020.92011

Received: February 5, 2020

Accepted: March 6, 2020

Published: March 9, 2020

Copyright $\odot 2020$ by author(s) and Scientific Research Publishing Inc. This work is licensed under the Creative Commons Attribution International License (CC BY 4.0).

http://creativecommons.org/licenses/by/4.0/

\begin{abstract}
Background: This study aimed to determine the safety and efficacy of sphenopalatine artery (SPA) surgery in patients with refractory epistaxis and to identify factors associated with the indications for surgery to assist clinicians in making prompt and appropriate decisions regarding SPA surgery for refractory epistaxis. Methods: We analyzed 97 consecutive patients with nasal bleeding hospitalized in our institution between April 2009 and March 2018. Clinicopathological characteristics and clinical outcomes were analyzed from retrospective chart reviews. Results: Of the 97 patients, 28 ( 29 sides) required SPA surgery. There were no differences in sex, anti-coagulant drug or antiplatelet use, hemoglobin concentration, or platelet count between Group A (patients who required surgery) and Group B (patients who did not require surgery). Age (58 vs. 67 years, $p=0.038$ ) and severity of comorbidity ( 0 vs. 1 , $p=0.039$ ) were significantly lower in the surgery group. Patients who were younger and had lower Charlson Comorbidity Index (CCI) scores had more requirements for surgery than those who were older and had higher CCI scores. There was no significant difference between the groups with respect to the proportion of patients receiving blood transfusions; however, the length of hospitalization was significantly longer in Group A than in Group B (8.9 vs. 8 days, $p=0.038$ ). Success rate (non-rebleeding rate) was $89 \%$, comparable to that reported in previous studies. Conclusions: Endoscopic SPA surgery was found safe and effective method with few complications. Younger patients with lower CCI scores were found appropriate for SPA surgery for refractory epistaxis due in part to a lower risk of anesthesia.
\end{abstract}

\section{Keywords}

Epistaxis, Sphenopalatine Artery, Emergency, Admission, Cauterization, Ligation, Endoscope 


\section{Introduction}

Idiopathic epistaxis is a primary emergent disease in otorhinolaryngology. Generally, anterior epistaxis is easy to control with observation or anterior gauze packing, whereas posterior epistaxis is often refractory to conservative treatment (i.e., anterior or posterior nasal packing) and sometimes recurs. Internal maxillary artery or external carotid artery operations without an endoscope for nasal bleeding have been performed [1]. Internal maxillary artery surgery requires a Caldwell-Luc procedure to be performed as the first step, which results in cheek pain in many cases. External carotid artery surgery requires an open neck procedure, which involves neck incision with possible sacrifice of other important arteries such as the facial and lingual arteries. However, as the techniques for endoscopic nasal paranasal surgery have advanced owing to improvements in endoscopic and surgical devices, endoscopic sphenopalatine artery (SPA) surgery for intractable epistaxis has become recognized as an alternative to the open neck procedure.

In most cases of refractory epistaxis, the responsible artery is the SPA, followed by the anterior ethmoid artery (AEA) [2] [3] [4] [5]. Previous studies have reported that endoscopic SPA surgery is a safe and effective treatment for repeated and refractory nasal bleeding [1] [5] [6] [7] [8]. Nevertheless, a strategy for selecting suitable candidates for SPA surgery has not yet been established. We searched for factors that may lead to the requirement of surgery for nasal bleeding in patients. In some cases, nasal packing might be as effective as an invasive treatment. By employing nasal packing, surgery can be avoided. The method also has less risk of complications than surgery, despite the pain and risk of bleeding associated with packing insertion and removal [9]. The endovascular approach (embolization) is also an option for refractory nasal bleeding. Embolization is a less invasive technique compared to surgery and is mostly performed under locoregional anesthesia. However, the procedure is associated with such severe complications as blindness, stroke, and facial paralysis [9]. In our retrospective study, we investigated the safety and efficacy of SPA surgery at a regional core hospital and identified factors associated with the need for surgery.

\section{Methods}

\subsection{Indication and Inclusion Criteria for Admission and SPA Surgery}

Inclusion criteria for admission were as follows: 1) frequent epistaxis of unknown origin seen at our institution, 2) cases of rebleeding transferred from other medical clinics, 3) anemia requiring transfusion, and 4) a history of anticoagulant or antiplatelet drug use. Other patients included in our study were observed in the outpatient clinic in our institution with or without nasal packing. In cases of rebleeding upon admission or when seen at the outpatient clinic, we performed endoscopic SPA surgery, whereas in cases where SPA surgery was contraindicated, we performed embolization. 


\subsection{Patients and Data Extraction}

A retrospective chart review was performed of all patients who were admitted to our institution for the treatment of intractable epistaxis between April 2009 and March 2018. The exclusion criteria were as follows: 1) epistaxis from nasal and paranasal tumors and epistaxis following cancer treatment, 2) epistaxis resulting from aneurysm treated with endovascular embolization, and 3) no routine blood examination data available. A total of 105 patients with intractable epistaxis treated in in-hospital settings who underwent the conservative treatment were identified in our hospital registry. After applying the inclusion and exclusion criteria, 97 patients were finally included in our analysis. SPA surgery was planned for patients resistant to conservative treatment. Clinicopathological characteristics (sex, age, anti-coagulant drug or antiplatelet use, comorbidity, hemoglobin concentration, and platelet count at presentation) and clinical outcomes (transfusion, hospitalization period, and rebleeding) were analyzed. Severity of comorbidity was determined according to the Charlson Comorbidity Index (CCI) [10].

The study protocol was approved by the Institutional Review Board of the Osaka General Medical Center (Osaka, Japan). Research was conducted in accordance with the 1964 Declaration of Helsinki and its later amendments. Given the retrospective nature of the study, the need for informed consent was waived by the institutional review board in accordance with the ethical guidelines for epidemiological research formulated by the Ministry of Health, Labor and Welfare of Japan.

\subsection{Surgical Procedure}

We searched the nasal cavity for bleeding sites using a rigid endoscope. if a bleeding site was found, we coagulated the site. Otherwise, we performed SPA ligation or cauterization. A vertical incision in front of the posterior margin of the maxillary sinus was made from the horizontal portion of the ground lamella to the insertion of the inferior turbinate on the lateral nasal wall, and a mucosal flap was elevated. Subsequently, the medial side of the ethmoidal bony crest was partially removed. The ethmoidal bony crest is formed by the conjunction between the maxillary and palatine bones. The maxillary sinus may be the landmark to clarify the location of the ethmoidal bony crest. The sphenopalatine foramen was visualized, and the SPA was identified. The SPA sometimes has several branches [11] [12] [13] [14]. In such cases, we try to address all branches of the SPA, if possible. Ligation or cauterization was performed at the surgeon's discretion. Cauterization was performed using an active device for ultrasonic treatment. Ligation was performed using two small metal clips placed on the artery. Oxidized regenerated cellulose was placed at the sphenopalatine foramen, and the flap was replaced.

\subsection{Statistical Analysis}

Relationships between categorical variables were assessed using the chi-square 
test or Fisher's exact test, and those between categorical and continuous variables were assessed using the Wilcoxon test. The Cochrane-Armitage test was used to assess the association between categorical and ordinal variables. The probability $(p)$ value $<0.05$ was considered statistically significant. JMP version 14 statistical software (SAS Japan, Tokyo, Japan) was used to perform all statistical analyses.

\section{Results}

A total of 97 patients (61 men and 36 women) were enrolled in this retrospective study. The median age was 66 years, and age range was 10 - 95 years. Twenty-eight patients received anticoagulant or antiplatelet drugs. Twelve patients underwent transfusion. The average length of hospitalization stay was $8.3 \pm 6.6$ days. The number of rebleeding cases after admission with conservative treatment was 31 . Surgery was necessary and performed in 28 patients, and nasal re-packing was performed in 3 patients.

There were 28 patients ( 19 men and 9 women) who required surgery (Group A). Surgery was performed on both sides in one patient. The median age was 58 years, and the age range was 16 - 80 years. Six patients received anticoagulant or antiplatelet drugs. Five patients received transfusions. The median length of hospitalization stay was $8.9 \pm 4.4$ days. The number of bleeding points for cauterization was 5 sides, and cauterization of SPA was performed on 11 sides and ligation of SPA on 13 sides. Twenty-five patients had no nasal bleeding postoperatively. Three patients had recurrent epistaxis postoperatively. The postoperative rebleeding rate was $11 \%$. We performed re-operation, embolization, and gauze packing as salvage treatment in these cases. Bilateral SPA surgery was performed in one patient because the bleeding sides were unknown. Only one patient required both SPA and AEA surgery. This patient was suspected of having nasal bleeding from the region fed by the AEA. There was no rebleeding after SPA and AEA surgery.

There were 69 patients who did not require surgery (Group B, 42 men and 27 women). The median age was 67 years, and the age range was 10 - 95 years. Twenty-two patients received anticoagulant or antiplatelet drugs. Seven patients underwent transfusion. The average of hospitalization stay was $8 \pm 7.2$ days.

\section{Comparison between Patients Who Required Surgery (Group A) and Those Who Did Not (Group B)}

There were no differences with respect to sex, anticoagulant use, hemoglobin concentration, and platelet count between the groups. There were significant differences in the groups with regard to age (group A: 58, group B: 67, $p=0.038$ ) and CCI (group A: 0, group B: $1, p=0.039$ ) (Table 1).

The proportion of patients requiring blood transfusion was not significantly different between the groups ( $p=0.979)$; however, the length of hospitalization was significantly longer in Group A than in Group B ( 8.9 vs. 8 days, $p=0.038$ ) (Table 2). 
Table 1. Patient characteristics.

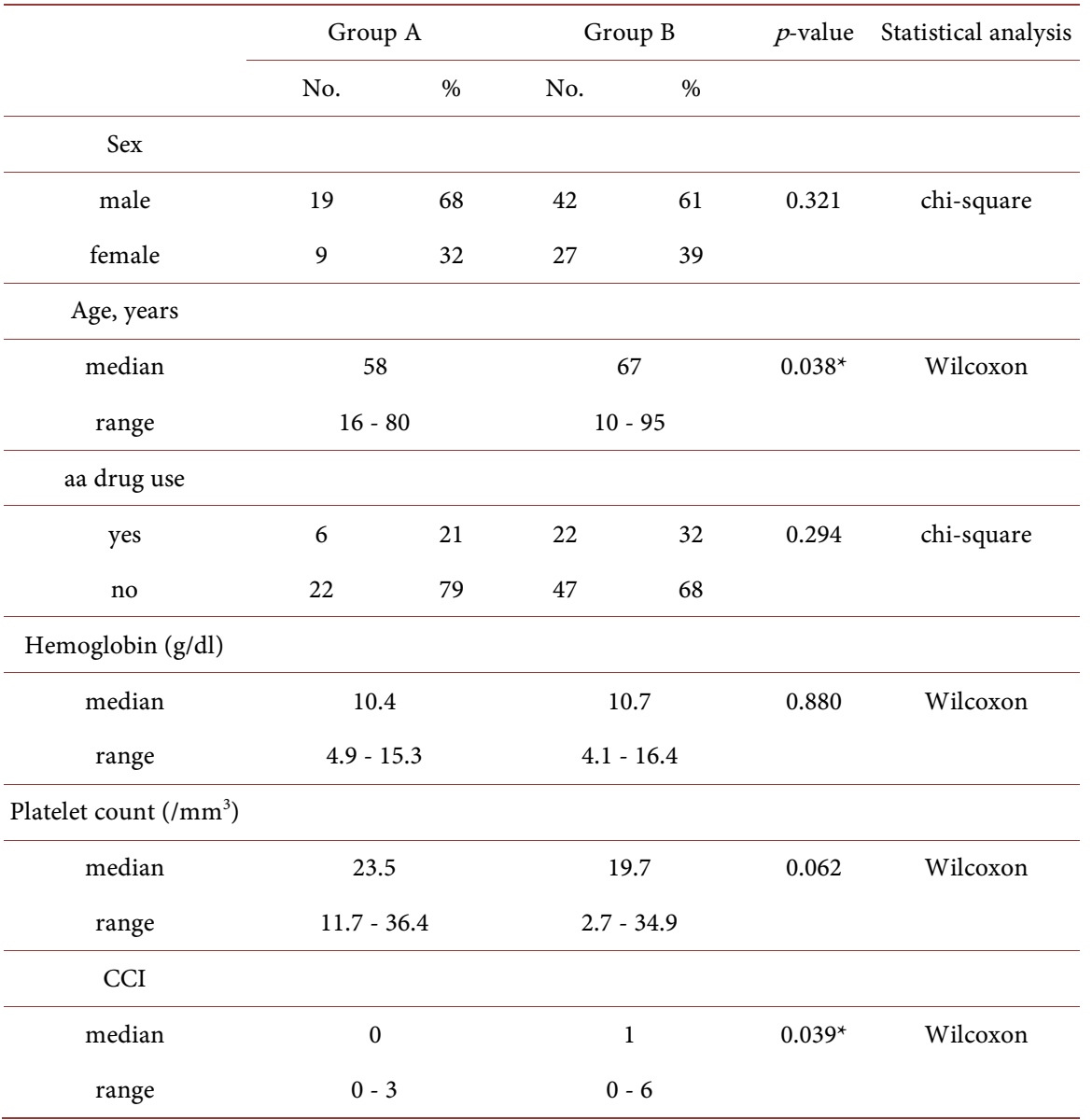

Abbreviation: CCI, Charlson Comorbidity Index, aa drug use, anti-coagulant drug or antiplatelet use.

Table 2. Treatment results.

\begin{tabular}{|c|c|c|c|c|c|}
\hline & \multicolumn{2}{|c|}{ Group A } & \multicolumn{2}{|c|}{ Group B } & \multirow[t]{2}{*}{$\mathrm{p}$ value } \\
\hline & No. & $\%$ & No. & $\%$ & \\
\hline \multicolumn{6}{|c|}{ rebleeding } \\
\hline & 3 & 11 & & & \\
\hline \multicolumn{6}{|c|}{ transfusion } \\
\hline yes & 5 & 17 & 7 & 10 & 0.979 \\
\hline no & 23 & 83 & 62 & 90 & \\
\hline \multicolumn{6}{|c|}{ hospitalization days } \\
\hline median & \multicolumn{2}{|c|}{8.9} & \multicolumn{2}{|c|}{8} & $0.038^{*}$ \\
\hline range & \multicolumn{2}{|c|}{$3-23$} & \multicolumn{2}{|c|}{$1-41$} & \\
\hline
\end{tabular}

Abbreviation: CCI, Charlson Comorbidity Index.

\section{Discussion}

Epistaxis is commonly seen in ENT clinics and is classified as anterior or posterior bleeding. In many cases, anterior nasal bleeding occurs from the Kiessel- 
bach's plexus anatomically [15]. This bleeding area is easy to treat in many cases due to the ease of visibility and accessibility of the area when using surgical tools. In contrast, in posterior nasal bleeding, the bleeding area is difficult to identify. The majority of the posterior blood supply of the nasal cavity is provided by the SPA [13] [15]. These findings suggest that endoscopic SPA surgery is effective for posterior epistaxis [1]-[8] [11] [14] [16] [17]. Background factors and characteristics of patients suitable for this surgery have not been clearly identified. In the present study, we aimed to determine whether surgical procedures were required or if conservative treatment should be continued in our patients. The theoretical underpinning was that if SPA surgeries were performed in a timely fashion, gauze packing or observational admissions may be unnecessary. Younger patients have fewer risks of anesthesia, as do those with lower CCI scores. In our retrospective study, patients of younger ages and those with lower CCI scores tended to receive SPA surgery. There were no differences with respect to sex, anticoagulant use, hemoglobin concentration, and platelet count between the groups, thus these factors were not related to the requirement of surgery in our study.

The surgical success rate was $89 \%$ ( $25 / 28$ cases); this result is comparable to that obtained in previous studies [1] [2] [5] [6] [7] [8].

Bilateral SPA surgery is sometimes necessary when the affected sides of nasal bleeding are unknown. In our study, bilateral SPA surgery was performed in only one patient. When posterior nasal bleeding is aggressive, most blood or coagulated material falls into the nasopharynx. In these situations, it is difficult to identify the bleeding sites. Septal deviation and hypertrophic turbinates often prevent visualization of the bleeding regions [2].

The main vessels that supply the nasal mucosa are the SPA and AEA [12] [16]. In our study, there were no cases of AEA surgery alone without SPA surgery. SPA and AEA surgery were both performed in only one patient. In that patient, suspicious bleeding sites appeared to be in the region fed by the AEA. Therefore, we performed AEA surgery in addition to SPA surgery. Nevertheless, we could not evaluate the effect of AEA surgery alone. In previous reports, AEA surgery alone for nasal bleeding was controversial. Asanu et al. [2] found that the success rates of SPA and AEA surgery versus AEA surgery alone were not statistically different.

The overall success rate of endovascular treatment in terms of adequately stopping nasal bleeding is comparable to that of surgery [17]. The endovascular approach is usually a less invasive technique than surgery, mostly performed under locoregional anesthesia. However, embolization is associated with such severe complications as blindness, stroke, and facial paralysis [9]. These could occur in cases of significant anastomosis between the vascular territory of the nose and the eye or brain [15]. Embolization is performed only on the arteries of the external carotid branches. Branches of the internal carotid arteries involved in nasal bleeding are the anterior and posterior ethmoid artery. Embolization for bleeding in the region of the ethmoidal arteries is a relative contraindication be- 
cause of the high risk of visual impairment.

Our basic strategy for refractory epistaxis is 1) conservative treatment such as nasal packing, 2) endoscopic SPA surgery, and 3) in case of contraindication of SPA surgery, embolization.

\section{Limitation}

In general, embolization is effective for refractory epistaxis. Because our study aimed to investigate the efficacy of SPA surgery for epistaxis, the effect or adverse event of embolization was not researched.

\section{Conclusion}

Endoscopic SPA surgery is an effective method for the treatment of refractory epistaxis, with few complications. We achieved a success rate of $89 \%$. In our retrospective study, we performed the most surgeries for epistaxis on younger patients and those with lower CCI scores because of their lower anesthesia risks.

\section{Ethical Approval}

The study protocol was approved by the Institutional Review Board of the Osaka General Medical Center (Osaka, Japan). Research was conducted in accordance with the 1964 Declaration of Helsinki and its later amendments. Given the retrospective nature of the study, the need for informed consent was waived by the institutional review board in accordance with the ethical guidelines for epidemiological research formulated by the Ministry of Health, Labor and Welfare of Japan.

\section{Conflicts of Interest}

The authors declare no conflicts of interest regarding the publication of this paper.

\section{References}

[1] Kitamura, T., Takenaka, Y., Takeda, K., Oya, R., Ashida, N., Shimizu, K., et al. (2019) Sphenopalatine Artery Surgery for Refractory Idiopathic Epistaxis: Systematic Review and Meta-Analysis. Laryngoscope, 129, 1731-1736. https://doi.org/10.1002/lary.27767

[2] Asanau, A., Timoshenko, A.P., Vercherin, P., Martin, C. and Prades, J.M. (2009) Sphenopalatine and Anterior Ethmoidal Artery Ligation for Severe Epistaxis. Annals of Otology, Rhinology \& Laryngology, 118, 639-644. https://doi.org/10.1177/000348940911800907

[3] George, A., Smatanova, K., Joshi, H., Jervis, S. and Oluwole, M. (2012) Sphenopalatine, Anterior Ethmoid and Internal Maxillary Artery Intervention in the Management of Refractory Epistaxis: Their Efficacy in 25 Patients. Clinical Otolaryngology, 37, 321-325. https://doi.org/10.1111/j.1749-4486.2012.02499.x

[4] Srinivasan, V., Sherman, I.W. and O'Sullivan, G. (2000) Surgical Management of Intractable Epistaxis: Audit of Results. Journal of Laryngology \& Otology, 114, 697-700. https://doi.org/10.1258/0022215001906732

[5] Nouraei, S.A., Maani, T., Hajioff, D., Saleh, H.A. and Mackay, I.S. (2007) Outcome 
of Endoscopic Sphenopalatine Artery Occlusion for Intractable Epistaxis: A 10-Year Experience. Laryngoscope, 117, 1452-1456.

https://doi.org/10.1097/MLG.0b013e318065b86f

[6] Wormald, P.J., Wee, D.T. and van Hasselt, C.A. (2000) Endoscopic Ligation of the Sphenopalatine Artery for Refractory Posterior Epistaxis. American Journal of Rhinology, 14, 261-264. https://doi.org/10.2500/105065800779954455

[7] Dutta, M. and Haldar, D. (2017) Optimizing the Outcome of Transnasal Endoscopic Sphenopalatine Artery Ligation in Managing Refractory Posterior Epistaxis: A Case-Control Analysis. Auris Nasus Larynx, 44, 554-560. https://doi.org/10.1016/j.anl.2016.10.008

[8] Snyderman, C.H., Goldman, S.A., Carrau, R.L., Ferguson, B.J. and Grandis, J.R. (1999) Endoscopic Sphenopalatine Artery Ligation Is an Effective Method of Treatment for Posterior Epistaxis. American Journal of Rhinology, 13, 137-140. https://doi.org/10.2500/105065899782106805

[9] Willems, P.W., Farb, R.I. and Agid, R. (2009) Endovascular Treatment of Epistaxis. American Journal of Neuroradiology, 30, 1637-1645. https://doi.org/10.3174/ajnr.A1607

[10] Charlson, M., Szatrowski, T.P., Peterson, J. and Gold, J. (1994) Validation of a Combined Comorbidity Index. Journal of Clinical Epidemiology, 47, 1245-1251. https://doi.org/10.1016/0895-4356(94)90129-5

[11] Agreda, B., Urpegui, A., Ignacio Alfonso, J. and Valles, H. (2011) Ligation of the Sphenopalatine Artery in Posterior Epistaxis. Retrospective Study of 50 Patients. Acta Otorrinolaringológica Española, 62, 194-198. https://doi.org/10.1016/j.otorri.2010.11.005

[12] Ram, B., White, P.S., Saleh, H.A., Odutoye, T. and Cain, A. (2000) Endoscopic Endonasal Ligation of the Sphenopalatine Artery. Rhinology, 38, 147-149.

[13] Holzmann, D., Kaufmann, T., Pedrini, P. and Valavanis A. (2003) Posterior Epistaxis: Endonasal Exposure and Occlusion of the Branches of the Sphenopalatine Artery. European Archives of Oto-Rhino-Laryngology, 260, 425-428. https://doi.org/10.1007/s00405-003-0618-7

[14] Minni, A., Dragonetti, A., Gera, R., Barbaro, M., Magliulo, G. and Filipo, R. (2010) Endoscopic Management of Recurrent Epistaxis: The Experience of Two Metropolitan Hospitals in Italy. Acta Oto-Laryngologica, 130, 1048-1052. https://doi.org/10.3109/00016481003621538

[15] Nicolay, S., Van Der Zijden, T., Voormolen, M., d'Archambeau, O., Maes, J., De Belder, F., et al. (2015) The Endovascular Treatment of Epistaxis. Journal of the Belgian Society of Radiology, 99, 65-73. https://doi.org/10.5334/jbr-btr.954

[16] Kumar, S., Shetty, A., Rockey, J. and Nilssen, E. (2003) Contemporary Surgical Treatment of Epistaxis. What Is the Evidence for Sphenopalatine Artery Ligation? Clinical Otolaryngology and Allied Sciences, 28, 360-363. https://doi.org/10.1046/j.1365-2273.2003.00724.x

[17] Swords, C., Patel, A., Smith, M.E., Williams, R.J., Kuhn, I. and Hopkins, C. (2017) Surgical and Interventional Radiological Management of Adult Epistaxis: Systematic Review. Journal of Laryngology \& Otology, 131, 1108-1130. https://doi.org/10.1017/S0022215117002079 